delayed bleeding all requiring endoscopic hemostasis. No perforation was seen.

Conclusions This early experience with SRS2 shows colorectal submucosal dissections are feasible with an acceptable safety profile. Resection times for lesions $\leq 4 \mathrm{~cm}$ were relatively quick and microwave coagulation appeared to be effective in most cases.

\section{ATU-01 ENDOSCOPIC AMPULLECTOMY: HOW SAFE IS IT IN UK SETTINGS?}

Mohamed Abdelrahim*, Lazaros Varytimiadis, Asma Al-Kandari, Ejaz Hossain, Patrick Goggin, Pradeep Bhandari, Sophie Arndtz. Queen Alexandra Hospital-Portsmouth, Portsmouth, UK

\subsection{6/gutjnl-2019-BSGAbstracts. 14}

Introduction Endoscopic ampullectomy is a minimally invasive technique of treating non-invasive lesions involving the ampulla of Vater, and offers an alternative to major surgical intervention. In this study, we describe the safety and outcome of this procedure from a large, tertiary centre.

Methods Data were prospectively collected on an electronic database. Parameters related to ampullectomy outcome and complications were retrospectively analyzed.

Results A total of 37 cases were included in this analysis, between 2009 and 2018. Average age was 66. Female represented $59.4 \%$ of patients. Mean duration of follow up was 62.3 weeks. $23(62.1 \%)$ lesions were pure ampullary, and the remaining involved the duodenal wall as well. Lesions ranged from 5 to $80 \mathrm{~mm}$ in size, with average size of $21.58 \mathrm{~mm} .5$ patients $(13.5 \%)$ had familial adenomatous polyposis FAP. Pre resection histology confirmed HGD in one patient $(2.7 \%)$, and neuro-endocrine tumor NET in one case $(2.7 \%)$. Post resection histology showed focal adenocarcinoma in 1 patient $(2.7 \%)$.

Prophylactic PD stenting was performed in 26 cases (70.2\%), adjunctive APC used in 7 cases (18.9\%), and Submucosal injection in 27 cases (72.9\%). En-bloc resection was achieved in 16 (43.2\%), while piecemeal in 21 cases $(56.7 \%)$. Recurrence observed in 9/37 cases (24.3\%). Piecemeal resection and lack of submucosal injection were significantly associated with higher risk of recurrence $(\mathrm{P}=0.0485, \mathrm{P}=0.0471$, respectively). Early bleeding (including intra-procedural and bleeding within 24 hours of procedure) happened in 5 patients $(13.5 \%)$, delayed bleeding (after 24 hours) in 2 cases (5.4\%), all were successfully treated endoscopically. Only 1 patient $(2.7 \%)$ had Pancreatitis, and one patient developed ampullary stenosis post procedure. There was no report of perforation, need for emergency surgery or 30 day mortality in this series.

Abstract ATU-01 Table 1 Outcomes and complications following endoscopic ampullectomy in a single UK tertiary endoscopy unit (Total number of patients $=37$ )

\begin{tabular}{lll}
\hline Outcomes & En-bloc resection & \\
& $\begin{array}{l}\text { Piecemeal resection } \\
\text { Recurrence }\end{array}$ & $16(43.2 \%)$ \\
Complications & $21(56.7 \%)$ \\
Bleeding & Early & \\
& Delayed & $5(13.5 \%)$ \\
Pancreatitis & & $2(5.4 \%)$ \\
Ampullary Stenosis & & $1(2.7 \%)$ \\
Perforation & & $1(2.7 \%)$ \\
\hline
\end{tabular}

Conclusion Our series demonstrate the safety of this procedure in the UK setting. Piecemeal resection, and lack of submucosal injection seems to predict risk of recurrence, while size of the lesion did not significantly affect this outcome.

\section{ATU-02 A PROSPECTIVE, COMPARATIVE STUDY OF PLENVU ${ }^{\circledR}$, MOVIPREP ${ }^{\circledR}$ AND SENNA/CITRAMAG AS BOWEL PREPARATION FOR SCREENING COLONOSCOPY}

Angad Dhillon*, Sarah Marshall, Adam Humphries, Brian Saunders. Wolfson Unit for Endoscopy, St Mark's Hospital, London, UK

\subsection{6/gutjnl-2019-BSGAbstracts. 15}

Introduction Efficacy and patient acceptability of bowel preparation are key components in colorectal cancer screening (CRC) populations. Plenvu is a new, 1 litre, low volume PEGbased product indicated for bowel cleansing in adults prior to colonoscopy. Two Phase III studies have demonstrated superior colon cleansing efficacy, with comparable safety and tolerability versus standard preparations. ${ }^{1} 2$

Methods The aim was to assess the effectiveness and acceptability of Plenvu as compared to two different regimes for bowel preparation. A total of 68 consecutive patients attending day-case screening colonoscopy as part of the Bowel Cancer Screening Program were allocated either Plenvu (Norgine UK) ( $\mathrm{n}=31$ ), Moviprep (Norgine, UK) (n = 26) or senna and Citramag (Sanochemia UK) $(\mathrm{n}=11)$. An adapted split dosing regime was used for morning and afternoon appointments. Patients recorded their experience of the preparation on a questionnaire and one of two experienced endoscopists blinded to the preparation given, assessed bowel cleansing using the Boston Bowel Preparation Scale (BBPS). The volume of fluid aspirated from the colon (total amount in suction canister) and volume of fluid required for mucosal washing (including $50 \mathrm{ml}$ syringes and automated irrigation pump use) was measured during each procedure.

Results All patients completed their prescribed dose of bowel preparation. A total of $55 \%$ of patients reported Plenvu as pleasant in taste; this was comparable to both Moviprep (59\%) and senna/Citramag (45\%). Plenvu provided superior bowel cleanliness in each segment of the colon with an average total BBPS score of 7.5 out of 9 as compared to Moviprep (6.4) and senna/Citramag (6.0) $(p=0.006)$. The median volume of fluid aspirated from the colon was $400 \mathrm{mls}$ for all three bowel preparations $(p=0.96)$. The median volume required for mucosal washing was lowest in the Plenvu group (200 mls) compared to Moviprep (350 mls) and senna/Citramag $(250 \mathrm{mls})(\mathrm{p}=0.04)$.

Conclusions Plenvu appears to be an effective and palatable bowel preparation, providing superior cleanliness and a reduced requirement for washing. It is currently the lowest volume PEG based solution available and an important alternative for patients unable to tolerate higher volume preparations.

\section{REFERENCES}

1. Bisschops R, Manning J, Clayton LB, et al. Colon cleansing efficacy and safety with 1 L NER1006 versus 2. L polyethylene glycol plus ascorbate: a randomized phase 3 trial. Endoscopy 2018 Jul 19. 